\title{
Evaluación del estado ecológico de los ríos de la cuenca del río Ebro mediante el índice trófico de macrófitos IVAM-G (Índice de Vegetación Acuática Macroscópica)
}

\author{
Pedro Tomás ${ }^{1, *}$, Jose Luis Moreno ${ }^{2}$, Marina Aboal $^{3}$, Javier Oscoz ${ }^{4}$, Concha Durán ${ }^{5}$ y Patricia \\ Navarro 5 \\ ${ }^{1}$ Laboratorio de Ensayos Técnicos S.A. ENSAYA. Pol. Ind. Valdeconsejo C/Aneto parcela 8-A 50410 Cuarte de \\ Huerva (Zaragoza). Tlf. 976566875, Fax. 976566612 E-mail: biologia@ensaya.es \\ ${ }^{2}$ Centro Regional de Estudios del Agua, Universidad de Castilla-La Mancha, Albacete. \\ ${ }^{3}$ Departamento de Biología Vegetal, Universidad de Murcia, Murcia. \\ ${ }^{4}$ Departamento de Biología Ambiental. Universidad de Navarra, Pamplona. \\ 5 Área de Calidad de Aguas. Confederación Hidrográfica del Ebro, Zaragoza. \\ * Corresponding author: biologia@ensaya.es
}

Received: 19/03/2015 Accepted: 14/12/2015

\section{RESUMEN}

Evaluación del estado ecológico de los ríos de la Cuenca del Ebro mediante el índice trófico de macrófitos IVAM-G (Índice de Vegetación Acuática Macroscópica)

La Directiva Marco del Agua 2000/60/CE propone a los macrófitos como indicadores biológicos para la evaluación del estado ecológico de los ríos. También establece la necesidad de definir las condiciones de referencia de dicho indicador para cada una de las tipologías de masas de agua superficial. En la Cuenca del Ebro se viene aplicando desde el año 2006 el índice de macrófitos IVAM-G, un índice trófico que otorga valores de tolerancia e indicador a una lista de taxones de flora acuática que incluye macroalgas, briófitos y plantas vasculares, con un nivel de resolución máxima de género. Con el fin de evaluar el estado ecológico de los ríos de la cuenca del Ebro, se han establecido las condiciones de referencia del IVAM-G y los valores de corte entre clases de estado ecológico para cinco tipos de ríos que cuentan con red de estaciones de referencia. Como modificación respecto al IVAM-G original, en este estudio se ha usado una escala de abundancia de 5 clases, por convergencia con la escala utilizada por el actual índice oficial en España, el IBMR. El IVAM-G ha mostrado una discriminación significativa entre estaciones impactadas y no impactadas y una correlación significativa $(p<0.001)$ con presiones antrópicas $(r=0.606)$, usos del suelo (agrícola, $r=0.503$ ), concentración de nutrientes (nitrito, $r=-0.605$; nitrato, $r=-0.594$; fosfato, $r=-0.47$ ) y con otros índices biológicos (IPS, diatomeas, $r=0.609$; IBMWP, macroinvertebrados, $r=0.506$ ). Adicionalmente, la respuesta obtenida por el IVAM-G fue similar a la obtenida por los índices biológicos incluidos en el RD 817/2015 (i.e., IBMWP e IPS) en la mayoría de los tipos de ríos, excepto en el tipo R-T26, donde hubo diferencias significativas con el IBMWP en la clasificación del estado ecológico de los tramos estudiados pero no con el IPS. Por todo ello, el IVAM-G es un índice que rinde unos resultados óptimos para el seguimiento del estado ecológico, simplificando a la vez el trabajo de campo y laboratorio respecto a otros índices de macrófitos con resolución taxonómica de especie. Sin embargo, es necesario realizar nuevas campañas de muestreo aplicando el índice IVAM-G junto con el IBMR y resto de índices de macrófitos nacionales, con el objetivo de poder comparar resultados, eficacias y costes.

Palabras claves: Directiva Marco del Agua, condiciones de referencia, índices biológicos, bioindicadores, calidad del agua.

\section{ABSTRACT}

Assessment of the ecological status of rivers of the Ebro Basin using the trophic macrophyte index IVAM-G (Macroscopic Aquatic Vegetation Index)

The Water Framework Directive 2000/60/EC proposes macrophytes as biological indicators for assessing the ecological status of rivers. It also indicates the need to establish reference conditions for that indicator in each of the types of surface water bodies. In the Ebro Basin, the macrophyte index IVAM-G has been applied since 2006, a trophic index that gives tolerance 
and indicator scores to aquatic taxa, including macroalgae, bryophytes and vascular plants, with a maximum taxonomic level of genus. In this study, we established the reference conditions and benchmarks of the IVAM-G for five river types which had a defined reference network. In this study, the only modification included respect to the original version of the IVAM-G, was the abundance scale that has been changed to 5 classes, in order to converge with the current Spanish official index IBMR. Testing the IVAM-G efficacy in the assessment, we obtained the following results: significant discrimination between impacted and non-impacted sites; strong and highly significant $(\mathrm{p}<0.001)$ response to pressure gradient $(\mathrm{r}=0.606) ;$ highly significant correlations with other biotic indices (IPS, diatoms, $\mathrm{r}=0.609 ;$ IBMWP, macroinvertebrates, $\mathrm{r}=0.506)$, land uses (agriculture, $\mathrm{r}=0.503$ ) and nutrients (nitrite, $\mathrm{r}=-0.605$; nitrate, $\mathrm{r}=-0.594$; phosphate, $\mathrm{r}=-0.47$ ). Additionally, the response obtained by the index IVAM-G was similar to that obtained by the indices included in RD 817/2015 (e.g. IBMWP and IPS) in most of the river classes except in the R-T26 type. In this river type the index IVAM-G showed significant differences with IBMWP in the classification of ecological status of the sections studied, however, with IPS no significant differences were observed. Therefore, the IVAM-G is an index that yields good results for monitoring the ecological status and, at the same time, it optimizes and simplifies the field work and laboratory processing. However, it is necessary to continue testing this index together with the current national indices in order to compare results, efficacy and costs.

Key words: Water Framework Directive, reference conditions, biological indices, bioindicators, water quality.

\section{INTRODUCCIÓN}

La Directiva Marco del Agua, 2000/60/CE (DMA en adelante), establece como uno de los indicadores de calidad biológicos para la clasificación del estado ecológico en los ríos, la composición y abundancia de los macrófitos. Además, señala la necesidad del "establecimiento de condiciones de referencia específicas del tipo para los tipos de masas de agua superficial" para los indicadores de calidad biológicos.

Los macrófitos son utilizados como indicadores de la calidad de las aguas, dada su sensibilidad a diferentes presiones, tanto a escala local como a escala de cuenca, así como a la eutrofización y la contaminación orgánica (Hering et al., 2006; Johnson \& Hering, 2009). Al mismo tiempo, son sensibles a cambios del hábitat a largo plazo, y pueden integrar en una escala temporal las perturbaciones (Westlake, 1975; Tremp \& Kohler, 1995). Los macrófitos también responden a los cambios hidromorfológicos (Baattrup-Pedersen \& Rins, 1999; Bernez et al., 2004, Schaumburg et al., 2004), mientras que los cambios en los usos del suelo en la cuenca de drenaje provocan variaciones en la composición y estructura de estas comunidades biológicas (Kuhar et al., 2007; Manolaki \& Papastergiadou, 2013; Zgola, 2014). Los macrófitos pueden utilizarse para evaluar diferentes presiones (Hering et al.,
2006; Marzin et al., 2012), por ejemplo, en el caso de vertidos orgánicos, la estructura y composición de estas comunidades bióticas se ha integrado en diferentes índices tróficos o de diversidad para evaluar la calidad del agua (Thiébaut et al., 2002). Su utilidad como indicadores biológicos depende del área de estudio y de los objetivos fijados en los programas de control ecológico (Resh, 2008). Sin embargo, diversos autores hacen una crítica generalizada de los índices tróficos europeos más utilizados, tanto en lo referente a su diseño como a su capacidad de respuesta en la detección de diferentes presiones (Demars et al., 2012; Demars \& Edwards, 2009; Willby et al., 2010). Así, la utilidad de los índices de macrófitos en la evaluación del estado ecológico de los ríos continúa actualmente cuestionándose, por lo que es necesario seguir realizando estudios con el fin de mejorar los métodos existentes, e incluso proponer otros nuevos que surjan con una base metodológica adecuada.

En España, se han aplicado en diferentes cuencas hidrográficas los siguientes índices nacionales: IM (Îndice de Macrófitos, Suárez et al., 2005) originado en la cuenca del río Segura; IVAM (Índice de Vegetación Acuática Macroscópica, Moreno et al., 2006) originado en la región de Castilla-La Mancha en sus dos versiones de resolución taxonómica: IVAM-G (género) e IVAM-B (familia); y, por último, la adaptación 
de dicho índice a las cuencas interiores catalanas (IVAM-FBL, Moreno et al., 2008). Recientemente, se ha desarrollado un nuevo índice denominado IMF (Índice de Macrófitos Fluviales, Cambra et al., 2012; Flor-Arnau et al., 2015), diseñado para ser aplicado globalmente al conjunto de ecotipos mediterráneos peninsulares. Entre los índices europeos, en España se ha utilizado experimentalmente el IBMR en la Cuenca del Ebro (Cambra et al., 2012), y también en el ámbito mediterráneo peninsular (Flor-Arnau et al., 2015).

Sin embargo, en la Instrucción de Planificación Hidrológica (Orden ARM/2656/2008) no se incluyeron los macrófitos para la evaluación del estado ecológico de los ríos, ya que en aquel momento no se disponía de datos suficientes sobre los diferentes índices aplicados a nivel nacional y, además, no se había realizado una intercalibración de índices de macrófitos a nivel europeo. Posteriormente, en el ejercicio de intercalibración de índices de macrófitos en ríos mediterráneos, se intercalibró el Índice Biológico de Macrófitos en Ríos, IBMR, (Haury et al., 2006; Comisión Europea, 2013; Aguiar et al., 2014), desarrollado en Francia, ya que los índices nacionales existentes en ese momento, el IM (Suárez et al., 2005) y la familia de índices IVAM (Moreno et al., 2006, 2008), presentaban diferencias metodológicas con el resto de índices europeos propuestos para el ejercicio de intercalibración (organismos recolectados, resolución taxonómica, clases de abundancia). Todo ello impidió la comparación de los datos entre países (fase "Intercalibration Feasibility Check" del proceso de intercalibración; Aguiar et al., 2014). Por último, recientemente se ha publicado el RD 817/2015 (MAGRAMA, 2015a), en el que se incluyen los macrófitos por primera vez para la evaluación del estado ecológico de los ríos, y se establecen condiciones de referencia y valores de corte entre clases de estado ecológico del IBMR para algunas tipologías de ríos mediterráneos.

El principal objetivo de este trabajo es presentar los resultados obtenidos al aplicar el índice trófico IVAM, en su versión con resolución a nivel de género, el IVAM-G (Moreno et al., 2006) en la cuenca del Ebro y evaluar su respuesta fren- te a presiones, comparándolo con el resultado de otros índices bióticos. Para ello, se abordan los siguientes objetivos específicos:

1. Proponer valores de referencia y de corte entre clases de estado ecológico para las principales tipologías fluviales de la cuenca del Ebro que cuentan con estaciones de referencia.

2. Evaluar la respuesta del IVAM-G frente a presiones antrópicas.

3. Comparar los resultados obtenidos por el IVAM-G con índices de otros indicadores biológicos.

4. Contrastar los resultados obtenidos al aplicar el IVAM-G con otros índices de macrófitos con resolución taxonómica de especie.

\section{METODOLOGÍA}

\section{Datos disponibles}

El IVAM-G lleva aplicándose en la cuenca del Ebro desde el año 2006. En este estudio se seleccionó la campaña de 2013, ya que fue el año para el cual existían mayor número de estaciones con datos disponibles simultáneamente para los tres indicadores biológicos a comparar: macroinvertebrados, diatomeas y macrófitos. Los muestreos de campo fueron realizados en todos los casos durante los meses de julio-octubre. Para los parámetros físico-químicos, en los casos en que se disponía de más de un dato anual por estación, se utilizó el valor promedio anual (Willby et al., 2010). Los muestreos y análisis fueron realizados siguiendo los siguientes protocolos: para macroinvertebrados Jáimez Cuellar et al. (2006) y CHE (2005a); para diatomeas CHE (2005b), UNE-EN 13946 (AENOR, 2004a), UNE-EN 14407 (AENOR, 2005a), MARM (2011a) y MAGRAMA (2013a); y para macrófitos CHE (2005c) y UNEEN 14184 (AENOR, 2004b).

Para evaluar el grado de correlación del IVAM-G con presiones antrópicas y con otros elementos de calidad se estimaron una serie de 
variables e índices. Las características físico-químicas del agua fueron medidas in situ con sensores multiparamétricos de $\mathrm{pH}, \mathrm{T}, \mathrm{O}_{2}$ y conductividad, mientras que para el resto de parámetros, $\mathrm{NO}_{3}, \mathrm{NO}_{2}, \mathrm{NH}_{4}, \mathrm{PO}_{4}, P_{\text {total }}$ y DQO, se tomaron muestras de agua que se conservaron en una nevera a $4{ }^{\circ} \mathrm{C}$ para ser analizadas en el laboratorio, siguiendo métodos estandarizados (APHA, 1989) y normas UNE: $\mathrm{NO}_{3}$ (4110-C), $\mathrm{NO}_{2}$ (UNE-EN 26777; 4500-NO $\left.\mathrm{N}_{2}-\mathrm{B}\right), \mathrm{NH}_{4}$ (4500$\mathrm{NH}_{4} . \mathrm{F}$ ), $\mathrm{PO}_{4}$ y $\mathrm{P}_{\text {total }}$ (4500-P. B; 4500-P. E; UNE-EN ISO 6878) y DQO (5220-D). Siguiendo las recomendaciones de la Directiva 2009/90 (Comisión Europea, 2009), para fines estadísticos se adoptó como valor de concentración la mitad del límite de cuantificación del método analítico para los resultados inferiores a dicho límite. Los índices hidromorfológicos calculados fueron el Índice de Hábitat Fluvial, IHF, (Pardo et al., 2002) y el Índice de Calidad del Bosque de Ribera, QBR (Munné et al., 1998; 2003). Los protocolos de cálculo de los índices biológicos fueron los siguientes: índice de macroinvertebrados Iberian Biomonitoring Working Party, IBMWP, (Alba Tercedor, 1988, 2002; MARM, 2011 b); índice de diatomeas, Índice de Poluosensibilidad Específica, IPS, (CEMAGREF, 1982; MAGRAMA, 2013b); y el índice de macrófitos IVAM-G (Moreno et al., 2006).

El índice IVAM-G, se diferencia de los índices de macrófitos IBMR e IMF en dos aspectos principales: 1) tiene una resolución taxonómica de género, 2) no incluye helófitos (plantas emer- gentes) en su listado. Sin embargo, los tres son índices tróficos que se basan en un sistema de puntuación de los taxones en función de su tolerancia o sensibilidad a la carga de nutrientes en el agua, en concreto de amonio y fosfato. También tienen en común la fórmula empleada para su cálculo (medias ponderadas; Zelinka \& Marvan, 1961). La fórmula de cálculo del IVAM-G fue la siguiente:

$$
I V A M-G=\sum v i_{i} * c_{i} * v t_{i} / \sum v i_{i} * c_{i}
$$

donde $v i_{i}$ es el valor indicador del taxón $i$, que oscila entre 1 y $2.5 ; c_{i}$ es el valor de cobertura del taxón $i$, representado por una escala de cinco valores: 1 (<0.1\%), $2(0.1-1 \%), 3(1-<10 \%), 4$ $(10-50 \%)$ y $5(>50 \%)$; y $v t_{i}$ el valor de tolerancia del taxón i, que varía entre 2 y 8 . Los valores vt e vi que se han aplicado corresponden a la versión original del IVAM-G (Moreno et al., 2006). Aquellos taxones recolectados que no figuraban en el listado original del IVAM-G, no se utilizaron en el cálculo del índice.

\section{Valores de referencia y límites EQR}

La red de referencia de la cuenca del Ebro tiene actualmente 37 estaciones repartidas en la siguientes tipologías recogidas en el RD 817/2015 (MAGRAMA, 2015a): R-T09, ríos mineralizados de baja montaña mediterránea $(n=2)$; RT11, ríos de montaña mediterránea silícea $(n=5)$;

Tabla 1. Condiciones de referencia del IVAM-G globales y para las 5 tipologías fluviales de la cuenca del Ebro con datos disponibles. R-T09: ríos mineralizados de baja montaña mediterránea; R-T11: ríos de montaña mediterránea silícea; R-T12: ríos de montaña mediterránea calcárea; R-T26: ríos de montaña húmeda calcárea; R-T27: ríos de alta montaña. (EQR = Ecological Quality Ratio). Global reference conditions for the index IVAM-G and for the five river types of the river Ebro basin with available data. R-T09: Mineralized Mediterranean low mountain rivers; $R$-T11: Siliceous Mediterranean mountain rivers; $R$-T12: Mediterranean calcareous mountain rivers; $R$-T26: Wet calcareous mountain rivers; $R-T 27:$ High mountain rivers $(E Q R=$ Ecological Quality Ratio $)$.

\begin{tabular}{|c|c|c|c|c|c|c|}
\hline $\mathrm{N}^{\mathrm{o}}$ estaciones ( $\mathrm{n}^{\mathrm{o}}$ datos $)$ & $\begin{array}{l}\text { Global } \\
37(98)\end{array}$ & $\begin{array}{c}\text { R-T09 } \\
2(5)\end{array}$ & $\begin{array}{c}\text { R-T11 } \\
5(15)\end{array}$ & $\begin{array}{l}\text { R-T12 } \\
15(39)\end{array}$ & $\begin{array}{l}\text { R-T26 } \\
10(28)\end{array}$ & $\begin{array}{c}\text { R-T27 } \\
4(10)\end{array}$ \\
\hline Mediana (cond. referencia) & 5.8 & 6.0 & 5.6 & 5.6 & 5.8 & 6.0 \\
\hline P 25 Límite MB/B & 5.4 & 5.9 & 5.6 & 5.3 & 5.3 & 5.7 \\
\hline EQR MB/B & 0.93 & 0.98 & 1 & 0.95 & 0.91 & 0.94 \\
\hline EQR B/Mo & 0.7 & 0.73 & 0.75 & 0.71 & 0.68 & 0.71 \\
\hline EQR Mo/Def & 0.47 & 0.49 & 0.5 & 0.47 & 0.46 & 0.47 \\
\hline EQR Def/M & 0.23 & 0.24 & 0.25 & 0.24 & 0.23 & 0.24 \\
\hline
\end{tabular}


$\mathrm{R}-\mathrm{T} 12$, ríos de montaña mediterránea calcárea $(n=15)$; R-T15, ejes mediterráneo-continentales poco mineralizados $(n=1)$; R-T26, ríos de montaña húmeda calcárea $(n=10)$; y R-T27, ríos de alta montaña $(n=4)$. A partir de los valores del índice IVAM-G obtenidos en las estaciones de referencia, se procedió al cálculo del valor correspondiente a las condiciones de referencia para cada tipología fluvial que contaba con al menos 5 datos disponibles. Con el objeto de reunir dicho mínimo de datos por tipología fluvial, ampliar y reforzar, en general, la significación estadística en todas las tipologías, se recurrió a los datos oficiales correspondientes a tres años (20112013). Tras calcular el valor medio del índice por estación, se determinó el valor de referencia en cada tipología fluvial mediante el cálculo de la mediana de dichos valores medios. Como valor límite o frontera entre el estado Muy Bueno/Bueno (MB/B) se consideró el percentil P25, (Comisión Europea, 2003; CHE, 2006). El valor de EQR (Ecological Quality Ratio) límite entre las clases $\mathrm{MB} / \mathrm{B}$ se calculó como:

$$
\mathrm{EQR}_{(\mathrm{MB} / \mathrm{B})}=\frac{\text { Límite MB } / \mathrm{B}(\mathrm{P} 25)}{\text { Valor de Referencia (Mediana) }}
$$

El resto de límites de corte entre clases de estado Moderado/Deficiente/Malo se calculó como:

$$
\mathrm{EQR}=\text { límite }(n+1)-((\mathrm{EQR} \mathrm{MB/B}) / 4))
$$

Posteriormente, se calcularon los EQR de los valores de IVAM-G para cada estación, dividiendo dicho valor por su correspondiente valor de re- ferencia. De la misma forma, para cada valor de los índices IBMWP e IPS fueron calculados los correspondientes valores de EQR, según los valores de referencia de dichos índices establecidos para cada tipología en el Anexo II del RD 817/2015 (MAGRAMA, 2015a). Teniendo en cuenta los valores de corte EQR entre clases de estado ecológico establecidos en dicha norma, se calculó el estado ecológico de las estaciones para cada uno de los índices.

\section{Estaciones impactadas y gradiente de presiones}

Para evaluar la capacidad de discriminación del IVAM-G entre estaciones impactadas y no impactadas, se realizó un test no paramétrico de Kruskal-Wallis (las variables no cumplían los criterios paramétricos) con el fin de comprobar la existencia de diferencias significativas entre los dos grupos. En total, se seleccionaron 91 estaciones que contaban con datos para los tres índices a comparar, de las cuales 55 correspondieron a la red de control operativo (impactadas) y 36 a la red de estaciones de referencia (no impactadas).

Para comprobar la respuesta de los indicadores a las presiones, se construyó un gradiente mediante un Análisis de Componentes Principales (PCA) realizado sobre las variables disponibles indicadoras de presiones, en este caso los usos del suelo y variables físico-químicas, concentración de nutrientes y DQO (Demanda Química de Oxígeno). Se eliminaron las estaciones con valores muy extremos (outliers) de alguna de las variables físicas y químicas $(n=6)$, y también las que presentaron ausencia de datos de alguna variable

Tabla 2. Test de Kruskal-Wallis comparando los valores de EQR de las estaciones de referencia e impactadas para los tres índices biológicos considerados. $\mathrm{H}$ es el estadístico calculado en el test y $p$ el nivel de significación. ns: no significativo; $*$ : $p<0.05$; $* *: p<0.01 ; * * * p<0.001$. Códigos de tipologías descritos en la Tabla 1. Kruskal-Wallis test comparing EQR values between reference and impacted sites for the three considered biological indices. $H$ is the statistic calculated and $\mathrm{p}$ is the significance level. ns: not significant; $*$ p < 0.05; **: $\mathrm{p}<0.01 ; * * *: \mathrm{p}<0.001$. Codes of river types as in Table 1.

\begin{tabular}{ccccccc}
\hline Tipología & EQR IBMWP & EQR IPS & & EQR IVAM-G \\
\hline & $\mathrm{H}$ & $p$ & $\mathrm{H}$ & $p$ & $\mathrm{H}$ & $p$ \\
Global & $\mathrm{H}(1, \mathrm{~N}=91)=46.1$ & $* * *$ & $\mathrm{H}(1, \mathrm{~N}=91)=42.64$ & $* * *$ & $\mathrm{H}(1, \mathrm{~N}=91)=36.74$ & $* * *$ \\
R-T09 & $\mathrm{H}(1, \mathrm{~N}=17)=5$ & $*$ & $\mathrm{H}(1, \mathrm{~N}=17)=4.36$ & $*$ & $\mathrm{H}(1, \mathrm{~N}=17)=4.06$ & $*$ \\
R-T12 & $\mathrm{H}(1, \mathrm{~N}=42)=16.4$ & $* *$ & $\mathrm{H}(1, \mathrm{~N}=42)=14.81$ & $* * *$ & $\mathrm{H}(1, \mathrm{~N}=42)=15.33$ & $* * *$ \\
R-T26 & $\mathrm{H}(1, \mathrm{~N}=23)=8.96$ & $* *$ & $\mathrm{H}(1, \mathrm{~N}=23)=14.08$ & $* * *$ & $\mathrm{H}(1, \mathrm{~N}=23)=4.21$ & $*$ \\
\hline
\end{tabular}


$(n=5)$. En total, para realizar el PCA se utilizaron 115 estaciones pertenecientes a las redes de referencia, vigilancia y operativo. La superficie (porcentaje) de los usos del suelo agrícola, forestal y urbano de cada subcuenca vertiente a los puntos estudiados, se calculó utilizando un MDT de $20 \times 20 \mathrm{~m}$ de resolución y la capa de usos del suelo del Corine Land Cover 2006, todo ello en el entorno del Sistema de Información Geográfica ArcGIS 10.1 (ESRI, 2012). Las variables fueron previamente transformadas logarítmicamente (excepto la transformación arcoseno para porcentajes de usos del suelo) y estandarizadas, para evitar cualquier efecto debido al uso de diferentes escalas numéricas.

\section{Análisis de correlación y comparación de estado ecológico}

El grado de correlación entre los índices biológicos y las variables ambientales indicadoras de presiones se exploró por medio de análisis univariantes entre cada par de variables mediante el coeficiente de correlación no paramétrico de Spearman.

Por último, se aplicaron Tests de Wilcoxon, a nivel global y por tipologías, para analizar las diferencias en la calificación del estado ecológico realizada por los tres indicadores biológicos IBMWP, IPS e IVAM-G. Los análisis estadísticos se realizaron con los programas Statistica 7.0 y Past 3.01 (Hammer et al., 2001).

\section{RESULTADOS}

\section{Condiciones de referencia y discriminación entre estaciones impactadas y de referencia}

Se calcularon condiciones de referencia para 5 tipologías (Tabla 1). Los valores de referencia más elevados correspondieron a las tipologías R-T09 (ríos mineralizados de baja montaña mediterránea) y R-T26 (ríos de montaña húmeda calcárea).

Los valores medianos de EQR obtenidos en las estaciones de referencia para los tres índices fueron mayores que los correspondientes a la red de control operativo (impactadas) (Fig. 1).
Tabla 3. Resultados del Análisis de Componentes Principales (PCA) realizado para obtener el gradiente de presiones. Se presentan los autovalores, \% de varianza absorbida por los dos primeros ejes y los valores de correlación de las variables ambientales con los factores. Results of Principal Component Analysis (PCA) performed to obtain the pressure gradient. Eigenvalues, \% cumulated variance by the first two axes and the correlation values between environmental variables and factors are showed.

\begin{tabular}{crr}
\hline & Factor 1 & Factor 2 \\
\hline Eigenvalue & 3.85 & 1.67 \\
\% varianza & 48.11 & 20.83 \\
DQO & -0.74 & -0.20 \\
amonio & -0.45 & -0.61 \\
nitrito & -0.74 & -0.36 \\
nitrato & -0.78 & 0.34 \\
fosfato & -0.69 & -0.55 \\
urbano & -0.59 & -0.12 \\
agrícola & -0.78 & 0.55 \\
forestal & 0.72 & -0.62 \\
\hline
\end{tabular}

Estas diferencias fueron significativas según el test Kruskal-Walis para los tres índices biológicos comparados, tanto a nivel global como para las tipologías R-T09 (ríos mineralizados de baja montaña mediterránea), R-T12 (ríos de montaña mediterránea calcárea) y R-T26 (ríos de montaña húmeda calcárea; Tabla 2). Los valores de EQR del índice de macrófitos IVAM-G presentaron unos valores medianos y una variabilidad más cercanos al índice IPS de diatomeas que al índice IBMWP de macroinvertebrados.

\section{Respuesta a las presiones}

Los dos primeros ejes del PCA explicaron un porcentaje alto de la varianza total $(68.9 \%)$, contribuyendo el Factor 1 con un 48.1\% (Tabla 3). El primer eje (Factor 1) fue la nueva variable que representó el gradiente de presiones, siendo sus coordenadas los valores que cuantifican dicho gradiente. La concentración de nitrato y fosfato, la carga orgánica (DQO) y el uso agrícola fueron las variables que más contribuyeron al primer componente (Factor 1).

En general, los tres índices presentaron correlaciones altamente significativas con todas las variables estudiadas excepto con el oxígeno disuelto, el IHF y el amonio (Tabla 4). Estas tres va- 
riables presentaron un gradiente débil o casi nulo en el conjunto de datos analizados, mostrando una escasa variabilidad entre las estaciones estudiadas. El IVAM-G presentó una correlación más elevada con el índice de diatomeas IPS $\left(r_{s}=\right.$
0.609, $p<0.001)$ que con el índice de macroinvertebrados IBMWP $\left(r_{s}=0.506, p<0.001\right)$. Los valores de correlación del IVAM-G con los nutrientes (nitrito, nitrato y fosfato) fueron elevados y altamente significativos $(p<0.001)$, muy
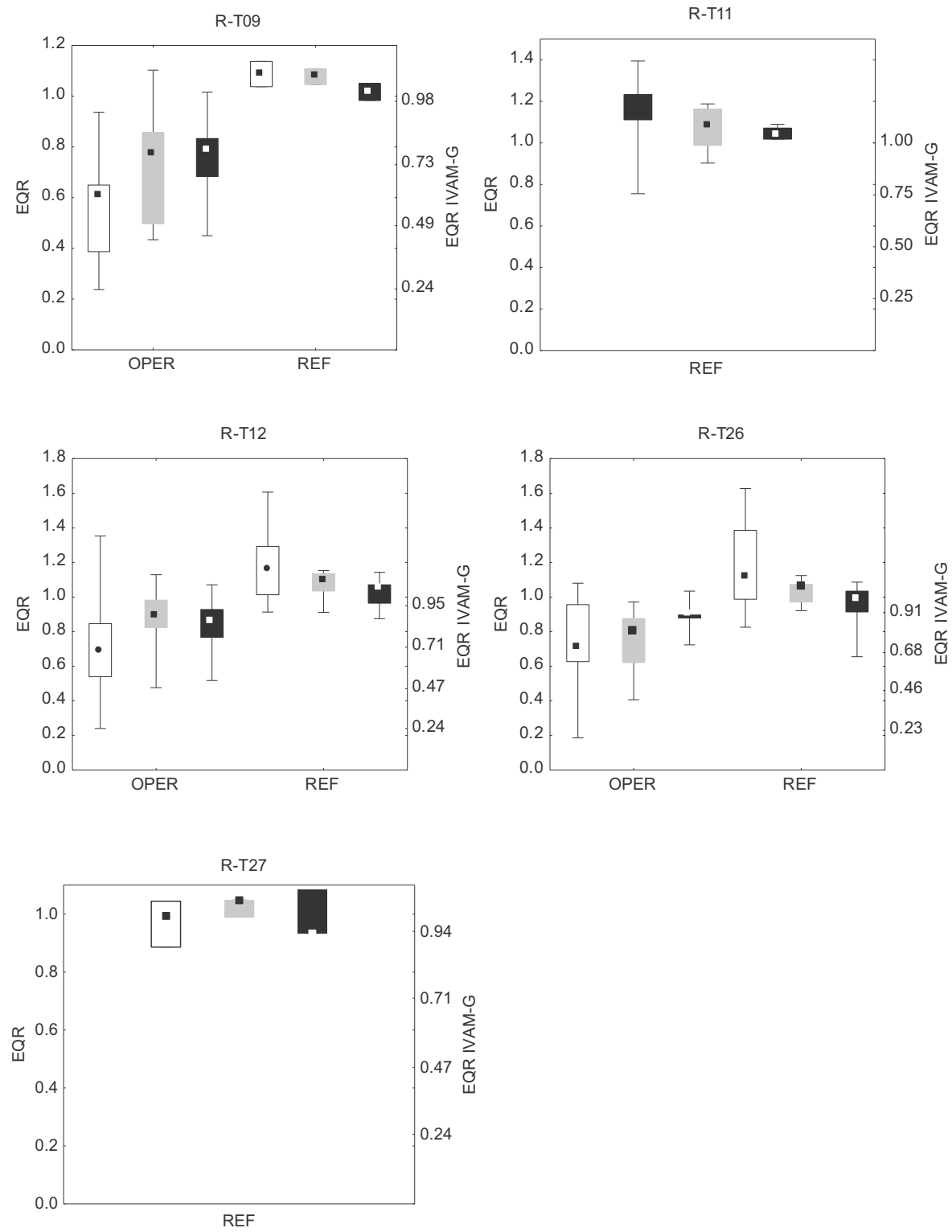

Figura 1. Valores de EQR (Ecological Quality Ratio) (mediana, percentiles 25-75, valor máximo-mínimo) correspondientes a las estaciones de control operativo y de referencia para los índices IBMWP (blanco), IPS (gris) e IVAM-G (negro) y las cinco tipologías estudiadas. En el eje derecho se presentan los límites entre clases de estado ecológico del índice IVAM-G para cada tipología. Oper: red control operativo; Ref: red de referencia. Códigos de tipologías y número de observaciones (n) en la Tabla 1. EQR values (median, percentiles 25-75, max-min values) for operational and reference sites for IBMWP (white), IPS (grey) and IVAM-G (black) indices and the five river types studied. On the right axis the boundaries between ecological status classes of IVAM-G index for each ecotype are showed. Oper: operational network; Ref: reference network. Codes of river types and number (n) as in Table 1. 
similares a las obtenidas por los otros dos índices bióticos (algo mayores que los obtenidos por el índice de macroinvertebrados IBMWP y ligeramente inferiores a los obtenidos por el índice de diatomeas IPS). Respecto a los usos del suelo, los valores de correlación con el uso agrícola y urbano también fueron elevados y similares a los alcanzados por el IPS e IBMWP.

\section{Estado Ecológico}

El mayor número de estaciones en estado muy bueno fue obtenido por el índice de diatomeas IPS (Fig. 2). El IVAM-G calificó el $60 \%$ de las estaciones en el mismo estado que el IPS, y el $48 \%$ en el mismo estado que el IBMWP, mientras que el IBMWP clasificó el $56 \%$ de las estaciones en el mismo estado que el IPS. El IVAMG calificó en estado Muy Bueno/Bueno al $97 \%$ de las estaciones de referencia, lo que supone el $97 \%$ de eficacia o exactitud en la calificación (dicho de otro modo, un $3 \%$ de error), mientras los índices IBMWP e IPS mostraron una exactitud del $100 \%$. En la red de control operativo el IBMWP obtuvo un $58 \%$ de las estaciones en estado Muy Bueno/Bueno, un $76 \%$ el IPS y un $84 \%$ el IVAM-G.
Mediante el test de Wilcoxon, se compararon las clases de estado ecológico obtenidas por cada uno de los indicadores, tanto a nivel global como por tipologías (Tabla 5). Se obtuvieron diferencias significativas entre los estados calificados por el IVAM-G y el IBMWP en la tipología RT26, y entre el IBMWP y el IPS en las tipologías R-T09 y R-T12. Ello indicaría que la calificación realizada por el IVAM-G mediante el uso de macrófitos podría considerarse similar a la realizada por el índice IPS de diatomeas, ya que entre ambos no existieron diferencias significativas.

\section{DISCUSIÓN}

En comparación con los macroinvertebrados o las diatomeas, los métodos de evaluación del estado ecológico de los ríos mediante la comunidad de macrófitos en España, se encuentran actualmente en una fase preliminar de aplicación y testado. Por ello, es de interés presentar los resultados obtenidos en las diferentes cuencas fluviales por los índices de macrófitos existentes, y al mismo tiempo comparar dichos resultados con otros índices biológicos. El IVAM-G es un índice adecuado para ser aplicado en la Cuenca del Ebro, ya

Tabla 4. Coeficientes de correlación de Spearman entre índices biológicos y las variables ambientales estudiadas. ns: no significativo; * $p<0.05$; ** $p<0.01 ; * * *: p<0.001$. Spearman correlation coefficients between biological indices and environmental variables. $n$ s, not significant; $* \mathrm{p}<0.05 ; * *: \mathrm{p}<0.01 ; * * *: \mathrm{p}<0.001$.

\begin{tabular}{|c|c|c|c|c|c|c|}
\hline & IVAM-G & $p$ & IPS & $p$ & IBMWP & $p$ \\
\hline IPS & 0.61 & $* * *$ & & & & \\
\hline IBMWP & 0.51 & $* * *$ & 0.54 & $* * *$ & & \\
\hline IHF & ns & & ns & & ns & \\
\hline QBR & 0.49 & $* * *$ & 0.53 & $* * *$ & 0.57 & $* * *$ \\
\hline Conductividad $20^{\circ}$ & -0.56 & $* * *$ & -0.54 & $* * *$ & -0.61 & $* * *$ \\
\hline $\mathrm{pH}$ & 0.23 & $*$ & 0.24 & $* *$ & 0.21 & $*$ \\
\hline Oxígeno disuelto & ns & & ns & & ns & \\
\hline DQO & -0.40 & $* * *$ & -0.61 & $* * *$ & -0.39 & $* * *$ \\
\hline Amonio & ns & & -0.26 & $* *$ & ns & \\
\hline Nitrito & -0.60 & $* * *$ & -0.62 & $* * *$ & -0.59 & $* * *$ \\
\hline Nitrato & -0.59 & $* * *$ & -0.53 & $* * *$ & -0.54 & $* * *$ \\
\hline Fosfato & -0.47 & $* * *$ & -0.55 & $* * *$ & -0.46 & $* * *$ \\
\hline Urbano & -0.45 & $* * *$ & -0.50 & $* * *$ & -0.58 & $* * *$ \\
\hline Agrícola & -0.55 & $* * *$ & -0.53 & $* * *$ & -0.62 & $* * *$ \\
\hline Forestal & 0.56 & $* * *$ & 0.53 & $* * *$ & 0.62 & $* * *$ \\
\hline Gradiente de presiones & 0.61 & $* * *$ & 0.69 & $* * *$ & 0.67 & $* * *$ \\
\hline
\end{tabular}


que discrimina significativamente entre estaciones de referencia y no referencia, presenta correlaciones elevadas y altamente significativas con los nutrientes, con el gradiente de presiones y con otros índices biológicos, especialmente con el índice de diatomeas IPS. Los valores de referencia y de corte propuestos en este estudio han resultado adecuados y pueden ser utilizados en el futuro. Los resultados obtenidos por otros indicadores biológicos incluidos en la IPH y el reciente RD 817/2015 (IBMWP e IPS) fueron similares a los obtenidos por el IVAM-G. Respecto a la comparación con otros índices de macrófitos se observa que, aunque la resolución taxonómica del IVAM$\mathrm{G}$ es de género, ha mostrado una eficacia similar, y en ciertos casos superior, a la obtenida por otros índices de macrófitos que alcanzan una resolución taxonómica a nivel de especie. De cualquier forma, con el fin de aumentar su eficacia, sería conveniente adaptarlo a la cuenca realizando dos modificaciones: 1) incluir los géneros que han sido recolectados en la cuenca del Ebro y que están ausentes en el listado original del IVAM-G; 2) adaptar los valores de tolerancia (vt) e indicador (vi) de los taxones a la cuenca.

\section{Condiciones de referencia}

Los valores de EQR que se obtuvieron para el índice IVAM-G discriminaron significativamente entre estaciones impactadas y no impactadas en las tipologías R-T09, R-T12 y R-T26. Estos resultados difieren de los obtenidos por el único estudio similar realizado en la cuenca del Ebro basados en la campaña de muestreo de 2009, donde el IVAM-G no mostró diferencias significativas entre estaciones impactadas y de referencia en dichas tipologías (Cambra et al., 2012). Por otra parte, la respuesta de los índices IBMWP e IPS fue similar al IVAM-G para esas tipologías, lo que nos indicaría la validez de los valores de referencia y de corte entre clases de estado ecológico calculados. El $97 \%$ de eficacia conseguido por el IVAM-G en la calificación de estaciones de referencia, se debe a que la estación de referencia 0816 (Esca/Burgui) se localiza aguas abajo de un puente y un azud, y presenta un sustrato inestable, condiciones que no permitieron el desarrollo de una comunidad de macrófitos correspondiente al estado Bueno o Muy Bueno (al contrario que los índices de macroinvertebrados y diatomeas).

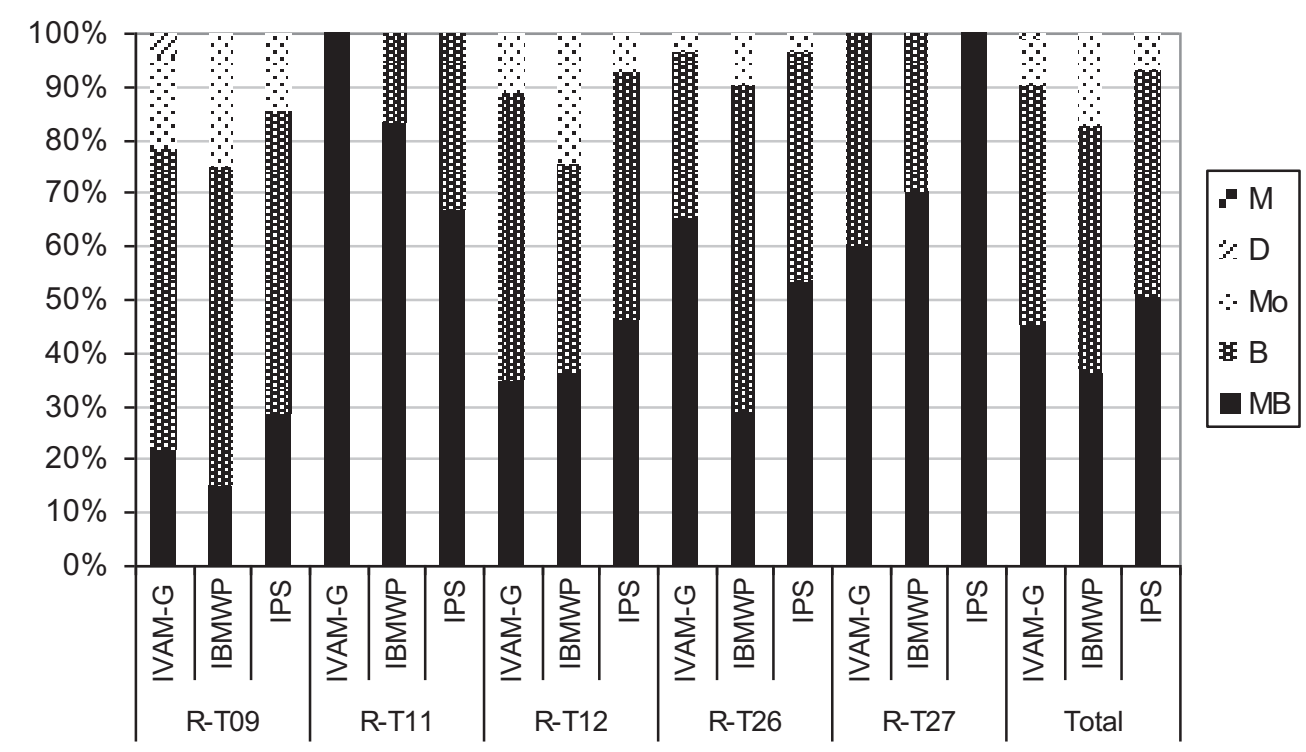

Figura 2. Estados ecológicos obtenidos por los índices biológicos para cada ecotipo fluvial y para el total de estaciones en 2013. MB: Muy Bueno; B: Bueno; Mo: Moderado; D: deficiente; M: Malo. Ecological status obtained by biological indices for each river ecotype and for all sites in 2013. MB: High; B: Good; Mo: Moderate; D: Poor; M: Bad. 
La capacidad de discriminación entre estaciones impactadas y no impactadas por el IVAM-G, también ha sido comprobada para numerosas tipologías mediterráneas a nivel peninsular, resultando similar a la del índice IMF (ambos discriminaron en 5 de los 8 tipos de ríos analizados) que utiliza una resolución taxonómica de especie (Flor-Arnau et al., 2015). Esta capacidad de discriminación tiene su importancia en el monitoreo y en la gestión, ya que la clasificación errónea del estado de una masa de agua, sobre todo si su estado es inferior a bueno, implica realizar un programa de medidas para que se recupere el buen estado.

Los valores de referencia del IVAM-G obtenidos en este estudio fueron en general inferiores a los obtenidos en trabajos anteriores (Moreno et al., 2006, Cambra et al., 2012). Esto puede deberse a que para establecer los valores de referencia en un tipo fluvial, es importante incluir un número suficiente de datos que recojan la variabilidad espacial y temporal dentro de los ecotipos. En los trabajos anteriormente citados, los valores de referencia se calcularon a partir de un único año, mientras que en el presente estudio se ha considerado una serie de tres años, teniendo en cuenta la variabilidad interanual debida a diferentes características hidrológicas en cuanto el régimen de precipitaciones y caudales circulantes.

\section{Respuesta a presiones}

Los valores de correlación del IVAM-G con la materia orgánica (DQO), los usos del suelo y los indicadores biológicos e hidromorfológicos fueron altamente significativos y en general superiores a los obtenidos anteriormente en la Cuenca del Ebro por el propio IVAM-G y otros índices de resolución taxonómica específica como el IBMR e IMF (ver informe de Cambra et al., 2012; ver Tabla S1 comparativa y Tabla S2, con las características generales de los índices, en material suplementario en www.limnetica.com). Respecto a los nutrientes, los valores de correlación con el fosfato y el nitrato obtenidos en el presente trabajo también fueron ligeramente superiores a los obtenidos en el citado informe, y a los obtenidos por Flor-Arnau et al. (2015) en el ámbito mediterráneo peninsular, tanto para el propio IVAM-G como para los índices de resolución taxonómica específica IMF e IBMR (Tabla S1). Sin embargo, y como suele ser habitual, el valor de correlación con el fosfato fue inferior al obtenido en su región de origen (Moreno et al., 2006). Es destacable la correlación del IVAM-G con la concentración de nitrato, que ha sido muy alta en comparación con otros estudios, incluso superior a la obtenida en su región de origen (Tabla S1). La débil correlación del IVAM-G, IPS e IBMWP con el amonio, como ya se ha comentado, ha sido debida a la falta de gradiente en los valores medidos: la mayoría de valores (el $80 \%$ aproximadamente) se encontraron por debajo del límite de cuantificación $\left(<0.13 \mathrm{mg} \mathrm{NH}_{4} / 1\right)$ del método de análisis utilizado en el laboratorio. Las estaciones de muestreo están alejadas de vertidos puntuales importantes. Únicamente se registraron valores altos de amonio en 6 estaciones situadas a 1-3 km aguas abajo de vertidos de depuradoras de poblaciones de más de 5000 habitantes. Esta débil correlación de los índices biológicos con el amonio en la red de control de la cuenca del Ebro, también se refleja en el informe realizado por Cambra et al. (2012; Tabla S1). Como norma general, es convenien-

Tabla 5. Test Wilcoxon realizado para detectar diferencias en la calificación del estado ecológico realizada por los diferentes indicadores, indicando la tiplogía donde la diferencia fue significativa (R-T12: ríos de montaña mediterránea calcárea). ns, no significativo; $*, p<0.05 ; * *, p<0.01 ; * * *, p<0.001$. Wilcoxon test performed to detect differences in the classification of ecological status by the different indicators. The river type with significant difference is indicated (R-T12: Mediterranean calcareous mountain rivers). ns, not significant; $* \mathrm{p}<0.05 ; * * \mathrm{p}<0.01 ; * * * \mathrm{p}<0.001$.

\begin{tabular}{|c|c|c|c|c|c|c|}
\hline & \multicolumn{2}{|c|}{ IVAM-G } & \multicolumn{2}{|r|}{ IBMWP } & \multicolumn{2}{|r|}{ IPS } \\
\hline & $\mathrm{Z}$ & Tipologías & $\mathrm{Z}$ & Tipologías & $\mathrm{Z}$ & Tipologías \\
\hline IVAM-G & & & $3.14 *$ & $(\mathrm{R}-\mathrm{T} 26 * *)$ & $0.033^{n s}$ & \\
\hline IBMWP & $3.14 *$ & $(\mathrm{R}-\mathrm{T} 26 * *)$ & & & $3.65 * * *$ & $(\mathrm{R}-\mathrm{T} 09 *, \mathrm{R}-\mathrm{T} 12 * *)$ \\
\hline IPS & $0.033^{n s}$ & & $3.65 * * *$ & $(\mathrm{R}-\mathrm{T} 09 *, \mathrm{R}-\mathrm{T} 12 * *)$ & & \\
\hline
\end{tabular}


te comprobar previamente la existencia de gradientes bien definidos en las variables ambientales que se van a correlacionar con los índices, ya que es un elemento clave en la interpretación correcta de los resultados de correlación y, por tanto, de la respuesta a los nutrientes.

El IVAM-G fue sensible al uso agrícola y urbano, presentando también correlaciones superiores a las obtenidas en otros estudios por índices de resolución taxonómica a nivel de especie (Tabla S1).

Por último, la calificación del estado ecológico realizada por el IVAM-G no fue significativamente diferente a la realizada por el IPS y solo parcialmente diferente a la realizada por el IBMWP (para el tipo R-T26 se obtuvieron diferencias significativas). Ello refuerza y valida en general la utilidad del índice en la evaluación del estado ecológico en la cuenca del Ebro. El porcentaje global de estaciones con un estado $\mathrm{MB} / \mathrm{B}$ en la cuenca del Ebro fue del $90 \%$ según el IVAM-G, un porcentaje cercano al obtenido anteriormente en la cuenca (82\%; Cambra et al., 2012) o a escala peninsular (85\%; Flor-Arnau et al., 2015).

\section{Perspectivas de los métodos de evaluación de la comunidad de macrófitos}

Las perspectivas inmediatas del uso de índices de macrófitos en España contemplan la aplicación del índice IBMR (MAGRAMA, 2015a y b), ya que, al ser el único índice de macrófitos intercalibrado a nivel europeo por parte de España (en su versión original), ha sido propuesto como el índice "oficial" de obligatoria aplicación. Los cortes propuestos para el IBMR en el ejercicio de intercalibración europeo (Comisión Europea, 2013) han sido modificados en el reciente RD 817/2015 (MAGRAMA, 2015a), en el que se proponen los valores de referencia y de corte entre clases de estado ecológico del nuevo IBMR adaptado a España. La versión propuesta en el protocolo actual (MAGRAMA, 2015b), establece una lista taxonómica sensiblemente reducida respecto al IBMR original (Tabla S2), e introduce cambios en las puntuaciones de algunos taxones. Esta nueva versión, parece tener un carácter "pro- visional", ya que en dicha norma se indica que "el indicador requiere mejorar el nivel de confianza, bien porque no está intercalibrado, bien porque requiere mejorar su adaptación a los tipos nacionales".

Respecto a la comparación de resultados obtenidos hasta ahora por el IBMR frente a los índices nacionales (analizados en Flor-Arnau et al., 2015), el IVAM-G obtuvo mejores resultados que el IBMR, tanto en lo referente a la capacidad de discriminación entre estaciones impactadas y no impactadas (el IBMR solo discriminó en 2 de los 8 tipos de ríos analizados) como en los valores de correlación obtenidos con variables ambientales de presión, índices biológicos, hidromorfológicos y gradiente de presiones (Tabla S1). Igualmente, el nuevo índice IMF ha presentado una eficacia muy superior al IBMR en todos los aspectos testados en dicho estudio.

En relación con la resolución taxonómica, ambos índices, IBMR e IMF, requieren la determinación específica de briófitos, plantas vasculares y algunas algas, lo que supone una formación adicional del personal que realiza actualmente los muestreos y determinaciones taxonómicas. De cara a la gestión, la aplicación de un índice con resolución taxonómica de género supone un considerable ahorro de tiempo y costes en formación de analistas, en el muestreo y procesado de muestras en el laboratorio, así como también una disminución en la incertidumbre de los resultados debida a las dificultades taxonómicas de algunos grupos. Dados los resultados obtenidos en este y otros estudios, se deduce que sería conveniente en las próximas campañas de muestreo aplicar los índices nacionales además del IBMR, con el fin de contrastar eficacias y costes originados.

Por último, es importante destacar que los resultados que se van obteniendo en los escasos estudios hasta ahora publicados en España, son todavía muy dispares y a veces contradictorios (como se ha comprobado también en el resto de Europa). Los resultados difieren en gran medida dependiendo del conjunto de datos analizados (diferentes años, cuencas, analistas, etc.), presentando un nivel de casuística elevado que se refleja en una falta de reproducibilidad a escala espacial 
y temporal, y finalmente, en una elevada incertidumbre y nivel de confianza de los métodos. Este aspecto, junto con el hecho de que no se disponga actualmente de un método adaptado a los ríos españoles y a la vez intercalibrado, indica que nos encontramos aún en una fase muy preliminar en el uso de índices de macrófitos como indicadores del estado ecológico de los ríos españoles.

\section{AGRADECIMIENTOS}

Este trabajo no hubiera podido realizarse sin los análisis realizados por el personal del Laboratorio de Calidad de Aguas de la Confederación Hidrográfica del Ebro, los resultados facilitados por Rosa Angulo, del área de Calidad de las Aguas y el personal del SAIH. Los análisis de las muestras de diatomeas fueron realizados por Núria FlorArnau y Jaume Cambra de la Universidad de Barcelona.

\section{BIBLIOGRAFÍA}

AENOR. 1994. Calidad del agua. Determinación de nitrito. Método de espectrofotometría de absorción molecular. (ISO 6777:1984). (Versión oficial EN 26777:1993). Asociación Española de Normalización y Certificación. Madrid.

AENOR. 2004a. Calidad del agua-Guía para el muestreo en rutina y el pretratamiento de diatomeas bentónicas. UNE EN-13946: 2004. Asociación Española de Normalización y Certificación. Madrid.

AENOR. 2004b. Calidad del agua-Guía para el estudio de los macrófitos en cursos de agua. UNE EN-14184: 2004. Asociación Española de Normalización y Certificación. Madrid.

AENOR. 2005a. Calidad del agua-Guía para la identificación, recuento e interpretación de muestras de diatomeas bentónicas de ríos. UNE EN-14407: 2005. Asociación Española de Normalización y Certificación. Madrid.

AENOR. 2005b. Calidad del agua. Determinación del fósforo. Método espectrométrico de molibdato de amonio. (ISO 6878: 2004). (Versión oficial UNE-EN ISO 6878: 2005). Asociación Española de Normalización y Certificación. Madrid.
AFNOR. 2003. Projet final NF T90-395 (Indice Biologique Macrophytique en Rivière (IBMR)). Association Française de Normalisation. Saint-Denis La Plaine.

AGUIAR, F. C., P. SEGURADO, G. URBANIČ, J. CAMBRA, C. CHAUVIN, S. CIADAMIDARO, G. DÖRFLINGER, J. FERREIRA, M. GERM, P. MANOLAKI, M. R. MINCIARDI, A. MUNNÉ, E. PAPASTERGIADOU \& M. T. FERREIRA. 2014. Comparability of river quality assessment using macrophytes: A multi-step procedure to overcome biogeographical differences. Science of The Total Environment, 476-477: 757-767.

ALBA-TERCEDOR, J. \& A. SÁNCHEZ-ORTEGA. 1988. Un método rápido y simple para evaluar la calidad biológica de las aguas corrientes basado en el de Hellawell (1978). Limnetica, 4: 51-56.

ALBA-TERCEDOR, J., P. JÁIMEZ-CUÉLLAR, M. ÁLVAREZ, J. AVILÉS, N. BONADA, J. CASAS, A. MELLADO, M. ORTEGA, I. PARDO, N. PRAT, M. RIERADEVALL, S. ROBLES, C. E. SÁINZ-CANTERO, A. SÁNCHEZ-ORTEGA, M. L. SUÁREZ, M. TORO, M R. VIDAL-ABARCA, S. VIVAS \& C. ZAMORA-MUÑOZ. 2002. Caracterización del estado ecológico de ríos mediterráneos ibéricos mediante el índice IBMWP (antes

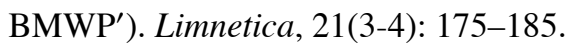

APHA. 1989. Standards methods for the examination of water and wastewater. 20th edition. American Public Health Association. Washington D.C.

BAATTRUP-PEDERSEN, A. \& T. RIIS. 1999. Macrophyte diversity and composition in relation to substratum characteristics in regulated and unregulated Danish streams. Freshwater Biology, 42: 375-385.

BERNEZ, I., H. DANIEL, J. HAURY \& M. T. FERREIRA. 2004. Combined effects of environmental factors and regulation on macrophyte vegetation along three rivers in Western France. River Research and Applications, 20: 43-59.

CAMBRA, J., N. FLOR-ARNAU, E. VELASCO \& C. VIEIRA. 2012. Red básica de macrófitos: resultados verano 2009 (Informe Técnico). Universitat de Barcelona-Confederación Hidrográfica del Ebro. 2012.

CEMAGREF. 1982. Etude des methods biologiques d'appréciation quantitative de la qualité des eaux. Rapport Q.E. Lyon, Agence de l'Eau Rhône-Méditerranée-Corse-Cemagref, Lyon.

COMISIÓN EUROPEA. 2000. Directiva 2000/60/CE del Parlamento Europeo y el Consejo de 23 de oc- 
tubre de 2000 por el que se establece un marco comunitario de actuación en el ámbito de la política de aguas (Directiva Marco del Agua). Diario Oficial de la Comunidad Europea, 327: 1-72.

COMISIÓN EUROPEA. 2003. WFD CIS. Guidance on establishing reference conditions and ecological status class boundaries for inland surface waters, REFCOND.

COMISIÓN EUROPEA. 2009. Directiva 2009/90/CE de la comisión de 31 de julio de 2009 por la que se establecen, de conformidad con la Directiva 2000/60/CE del Parlamento Europeo y del Consejo, las especificaciones técnicas del análisis químico y del seguimiento del estado de las aguas. Diario Oficial de la Unión Europea, 201: 36-38.

COMISIÓN EUROPEA. 2013. Decisión de la comisión del 20 de septiembre de 2013 por la que se fijan, de conformidad con la Directiva 2000/60/CE del Parlamento Europeo y del Consejo, los valores de las clasificaciones de los sistemas de seguimiento de los Estados miembros a raíz del ejercicio de intercalibración, y por la que se deroga la Decisión 2008/915/CE. Diario Oficial de la Unión Europea, 266: 1-47.

CONFEDERACIÓN HIDROGRÁFICA DEL EBRO. 2005a. Metodología para el establecimiento del Estado Ecológico según la Directiva Marco del Agua. Protocolos de muestreo y análisis para invertebrados bentónicos. Comisaría de Aguas, Confederación Hidrográfica del Ebro, Ministerio de Medio Ambiente.

CONFEDERACIÓN HIDROGRÁFICA DEL EBRO. 2005b. Metodología para el establecimiento del Estado Ecológico según la Directiva Marco del Agua. Protocolos de muestreo y análisis para Fitobentos (microalgas bentónicas). Comisaría de Aguas, Confederación Hidrográfica del Ebro, Ministerio de Medio Ambiente.

CONFEDERACIÓN HIDROGRÁFICA DEL EBRO. 2005c. Metodología para el establecimiento del Estado Ecológico según la Directiva Marco del Agua. Protocolos de muestreo y análisis para macrófitos. Comisaría de Aguas, Confederación Hidrográfica del Ebro, Ministerio de Medio Ambiente.

CONFEDERACIÓN HIDROGRÁFICA DEL EBRO. 2006. Establecimiento de condiciones de referencia y redefinición de redes en la cuenca del Ebro, segun la Directiva 2000/60/CE (expediente no 27/04-A) (Informe Técnico). Confederacion Hidrografica del Ebro-URS. Barcelona.
DEMARS, B. \& A. C. EDWARDS. 2009. Distribution of aquatic macrophytes in contrasting river systems: a critique of compositional-based assessment of water quality. The Science of the Total Environment, 407: 975-990.

DEMARS, B., J. POTTS, M. TRÉMOLIÈRES, G. THIÉBAUT, N. GOUGELIN \& V. NORDMANN. 2012. River macrophyte indices: not the Holy Grail!. Freshwater Biology, 57: 1745-1759.

ESRI. 2012. ArcGIS Desktop, 10.1. Redlands, CA: Environmental Systems Research Institute.

FLOR-ARNAU, N., M. REAL, G. GONZÁLEZ, J. CAMBRA, J. L. MORENO, C. SOLÀ \& A. MUNNÉ. 2015. Índice de Macrófitos Fluviales (IMF), una nueva herramienta para evaluar el estado ecológico de los ríos mediterráneos. Limnetica 34(1): 95-114.

HAMMER, Ø., D. A. HARPER \& P. D. RYAN. 2001. PAST: paleontological statistics software package for education and data analysis. Palaeontologia electronica, 4: 9.

HAURY, J., M. C. PELTRE, M. TRÉMOLIÈRES, J. BARBE, G. THIÉBAUT, I. BERNEZ, H. DANIEL, P. CHATENET, G. HAAN-ARCHIPOF, S. MULLER, A. DUTARTRE, C. LAPLACETREYTURE, A. CAZAUBON \& E. LAMBERTSERVIEN. 2006. A new method to assess water trophy and organic pollution-the Macrophyte Biological Index for Rivers (IBMR): its application to different types of river and pollution. Hydrobiologia, 570: 153-158.

HERING, D., R. K. JOHNSON, S. KRAMM, S. SCHMUTZ, K. SZOSZKIEWICZ \& P. F. M. VERDONSCHOT. 2006. Assessment of European rivers with diatoms, macrophytes, invertebrates and fish: a comparative metric-based analysis of organism response to stress. Freshwater Biology, 51: $1757-1785$.

JÁIMEZ CUELLAR, P., J. A. PALOMINO MORALES, J. M. LUZÓN ORTEGA \& J. ALBA TERCEDOR. 2006. Comparación de metodologías empleadas para la evaluación del estado ecológico de los cursos de agua. Tecnología del agua. 2006, 278: 42-57.

JOHNSON, R. K. \& D. HERING. 2009. Response of river inhabiting organism groups to gradients in nutrient enrichment and habitat physiography. Journal of Applied Ecology, 46: 175-186.

KUHAR, U., T. GREGORC, M. RENčELJ, N. ŠRAJKRŽIč \& A. GABERŠčI. 2007. Distribution of 
macrophytes and condition of the physical environment of streams flowing through agricultural landscape in north-eastern Slovenia. LimnologicaEcology and Management of Inland Waters, 37(2): 146-154.

MAGRAMA. 2013a. Protocolo de muestreo y laboratorio de flora acuática (organismos fitobentónicos) en ríos, $M L-R-D-2013$. Ministerio de Agricultura, Alimentación y Medio Ambiente. Código ML-RD-2013.

MAGRAMA. 2013b. Protocolo de cálculo del índice de poluosensibilidad específica, IPS-2013. Ministerio de Agricultura, Alimentación y Medio Ambiente. Código: IPS-2013

MAGRAMA. 2015a. Real Decreto 817/2015, de 11 de septiembre, por el que se establecen los criterios de seguimiento y evaluación del estado de las aguas superficiales y las normas de calidad ambiental. Boletín Oficial del Estado 219: 8058280669.

MAGRAMA. 2015b. Protocolo de cálculo del Índice Biológico de Macrófitos en ríos de España, IBMR2015. Ministerio de Agricultura, Alimentación y Medio Ambiente. Código: IBMR-2015.

MAGRAMA. 2015c. Protocolo de Muestreo y Laboratorio de Macrófitos en ríos, IBMR-2015. Ministerio de Agricultura, Alimentación y Medio Ambiente. Código IBMR-2015.

MANOLAKI, P. \& E. PAPASTERGIADOU. 2013. The impact of environmental factors on the distribution pattern of aquatic macrophytes in a middlesized Mediterranean stream. Aquatic Botany, 104: 34-46.

MARM. 2008. Orden ARM/2656/2008, de 10 de septiembre por la que se aprueba la Instrucción de Planificación Hidrológica. Ministerio de Medio Ambiente y Medio Rural y Marino. Boletín Oficial del Estado, 229: 38472-38582.

MARM. 2011a. Protocolo de muestreo y laboratorio de flora acuática (organismos fitobentónicos) en ríos vadeables, $M L-R-D-2011$. Ministerio de Medio Ambiente y Medio Rural y Marino. Código: ML-R-D-2011.

MARM. 2011b. Protocolo de cálculo del índice IBMWP, IBMWP-2011. Ministerio de Medio Ambiente y Medio Rural y Marino.Código: IBMWP2011.

MARZIN, A., V. ARCHAIMBAULT, J. BELLIARD, C. CHAUVIN, F. DELMAS \& D. PONT. 2012. Ecological assessment of running waters: Do macrophytes, macroinvertebrates, diatoms and fish show similar responses to human pressures?. Ecological Indicators, 23: 56-65.

MORENO, J. L., C. NAVARRO \& J. DE LAS HERAS. 2006. Propuesta de un índice de vegetación acuática (IVAM) para la evaluación del estado trófico de los ríos de Castilla-La Mancha: Comparación con otros índices bióticos. Limnetica, 25(3): 821-838.

MORENO, J. L., J. DE LAS HERAS, N. PRAT \& M. RIERADEVALL. 2008. Evaluación del estado trófico de tres cuencas interiores de Cataluña (Foix, Besòs y Llobregat) mediante la vegetación acuática: aplicación de un índice trófico (IVAM-FBL). Limnetica, 27(1): 107-118.

MUNNÉ, A., C. SOLÀ, M. RIERADEVALL \& N. PRAT. 1998. Índex QBR. Mètode per a l'avaluació de la qualitat dels ecosistemes de ribera. Estudis de la Qualitat Ecológica dels Rius. Diputació de Barcelona, Área de Medi Ambient. Barcelona.

MUNNÉ, A., N. PRAT, C. SOLÀ, N. BONADA \& M. RIERADEVALL. 2003. A simple field method for assessing the ecological quality of riparian habitat in rivers and streams: QBR index. Aquatic Conservation: Marine and Freshwater Ecosystems, 13: 147-163.

PARDO, I., M. ÁlVAREZ, J. CASAS, J. L. MORENO, S. VIVAS, N. BONADA, J. ALBATERCEDOR, P. JAIMEZ-CUÉLLAR, G. MOYÁ, N. PRAT, S. ROBLES, M. L. SUÁREZ, M. TORO \& M. R. VIDAL-ABARCA. 2002. El hábitat de los ríos mediterráneos. Diseño de un índice de diversidad de hábitat. Limnetica, 21(3-4): 115-133.

RESH, V. H. 2008. Which group is best? Attributes of different biological assemblages used in freshwater biomonitoring programs. Environmental Monitoring and Assessment, 138: 131-138.

SCHAUMBURG, J., C. SCHRANZ, J. FOERSTER, A. GUTOWSKI, G. HOFMANN, P. MEILINGER, S. SCHNEIDER \& U. SCHMEDTJE. 2004. Ecological classification of macrophytes and phytobenthos for rivers in Germany according to the water framework directive. Limnologica-Ecology and Management of Inland Waters, 34: 283-301.

SUÁREZ, M. L., A. MELLADO, M. M. SÁNCHEZMONTOYA \& M. R. VIDAL-ABARCA. 2005. Propuesta de un índice de macrófitos (IM) para evaluar la calidad ecológica de los ríos de la cuenca del Segura. Limnetica, 24(3-4): 305-318.

THIÉBAUT, G., F. GUÉROLD \& S. MULLER. 2002. Are trophic and diversity indices based on ma- 
crophyte communities pertinent tools to monitor water quality?. Water Research, 36: 3602-3610.

TREMP, H. \& A. KOHLER. 1995. The usefulness of macrophyte monitoring-systems, exemplified on eutrophication and acidification of running waters. Acta Botanica Gallica, 142(6): 541-550

WESTLAKE, D. F. 1975. Macrophytes. In Whitton B. A. (eds) River ecology, pp. 106-128, Barkley.

WILLBY, N., J. A. PITT \& G. PHILLIPS. 2010. The ecological classification of UK rivers using aquatic macrophytes. (Informe Técnico). Report SC010080/R1. Environment Agency, Bristol.

ZELINKA, M. \& P. MARVAN. 1961. Zur Präzisierung der biologischen Klassification der Reinheit flissender Gewässer. Archiv für Hydrobiologie, 57: 389-407.

ZGOLA, T. 2014. The response of aquatic plants to catchment land use for different types of lowland rivers. Applied ecology and environmental research, 12(1): 143-162. 
